\title{
Errata
}

\section{Application of Microwave Irradiation in the Synthesis of Carbohydrates}

S. K. Das* Synlett, 2004, 915.

An R group was left out of the structure of compound 47. The correct structure is shown below.

The author apologizes for this omission.

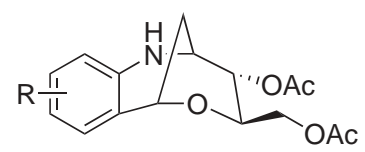

47 